\title{
Fatores de risco para incontinência urinária na mulher*
}

\author{
RISK FACTORS FOR URINARY INCONTINENCE IN WOMEN
}

FACTORES DE RIESGO PARA INCONTINENCIA URINARIA EN LAMUJER

\author{
Rosângela Higa', Maria Helena Baena de Moraes Lopes², Maria José dos Reis ${ }^{3}$
}

\section{RESUMO}

Trata-se de um estudo de revisão bibliográfica com o objetivo de identificar os principais fatores de risco ou associados à incontinência urinária (IU) na mulher. Utilizou-se a base de dados MEDLINE e LILACS e pesquisa em bibliotecas dos artigos publicados entre 1983 e 2003. Foram analisadas trinta e oito publicações em inglês e português, as quais relataram como os principais fatores de risco: idade, trauma do assoalho pélvico, fatores hereditários, raça, menopausa, obesidade, doenças crônicas, uso de alguns simpaticomiméticos e parasimpaticolíticos, constipação, tabagismo, consumo de cafeína e exercícios intensos na região abdominal. A enfermeira pode, por meio de anamnese, identificar estes fatores $e$ realizar intervenções para a prevenção e tratamento da IU, contribuindo para melhorar a qualidade de vida da mulher incontinente.

\section{DESCRITORES}

Incontinência urinária.

Fatores de risco.

Saúde da mulher.

Enfermagem.

\begin{abstract}
The goal of this study was to conduct a review of the main papers published between 1983 and 2003 on the main risk factors for urinary incontinence (UI) in women. Thirty-eight publications in English and Portuguese were analyzed using the MEDLINE and LILACS databases as well as through research in libraries. There is evidence that the main risk factors are age, pelvic floor trauma, hereditary factors, race, menopausal status, obesity, chronic diseases, use of some sympathomimetics and parasympatholitics, constipation, smoking, coffee consumption and intense abdominal exercises. Nurses are able to identify these factors through anamnesis and determine interventions for the prevention and treatment of UI, thus contributing to improve incontinent women's quality of life.
\end{abstract}

\section{KEY WORDS}

Urinary incontinence.

Risk factors.

Women's health.

Nursing.

\begin{abstract}
RESUMEN
Se trata de un estudio de repaso bibliográfico con objetivo de identificar los principales factores de riesgo o asociados a la incontinencia urinaria (IU) en la mujer. Se utilizó la base de datos MEDLINE e LILACS y pesquisa en bibliotecas de los artículos publicados entre 1983 e 2003 . Fueron analizadas treinta y ocho publicaciones en inglés y portugués, las cuales relataron como los principales factores de riesgo: edad, trauma de piso pélvico, factores hereditarios, raza, menopausia, obesidad, enfermedades crónicas, uso de algunos simpaticomiméticos y parasimpaticolíticos, constipación, tabaco, consumo de cafeína y ejercicios intensos en la región abdominal. La enfermera puede, por medio de la anamnesia, identificar estos factores y realizar intervenciones para la prevención y tratamiento de la IU, contribuyendo para mejorar la calidad de vida de la mujer incontinente.
\end{abstract}

\section{DESCRIPTORES}

Incontinencia urinaria. Factores de riesgo. Salud de la mujer. Enfermería.

\footnotetext{
* Extraído da Dissertação de Mestrado "Incontinência urinária: problema ocupacional entre profissionais de enfermagem", Departamento de Enfermagem, Faculdade de Ciências Médicas, Universidade Estadual de Campinas (FCM/UNICAMP), 2004. ${ }^{1}$ Enfermeira. Mestre em Enfermagem. Supervisora da Divisão de Enfermagem do Centro de Atenção Integral à Saúde da Mulher (CAISM), Universidade Estadual de Campinas (UNICAMP), Campinas, SP, Brasil. ro-higa@ig.com.br ${ }^{2}$ Enfermeira. Livre-Docente. Professora Associada do Departamento de Enfermagem, Faculdade de Ciências Médicas, Universidade Estadual de Campinas (FCM/UNICAMP), Campinas, SP, Brasil. mhbaena@fcm.unicamp.br ${ }^{3}$ Enfermeira. Especialista em Ginecologia. Supervisora da Divisão de Enfermagem do Centro de Atenção Integral à Saúde da Mulher (CAISM), Universidade Estadual de Campinas (UNICAMP), Campinas, SP, Brasil. mjreis03@hotmail.com.br
} 


\section{INTRODUCÃ̃}

A incontinência urinária (IU) é uma experiência que acomete milhões de pessoas de todas as idades, principalmente as do sexo feminino, afetando a qualidade de suas vidas. Com o aumento progressivo da expectativa de vida da população, o número de mulheres na meia idade tende a aumentar cada vez mais, sobretudo quando a IU torna-se mais prevalente ${ }^{(1)}$, amiúde, haverá um número crescente de casos e, muito deles não serão diagnosticados pela falta de busca no tratamento e por acreditarem que a IU é uma condição normal e resultado do processo de envelhecimento e não uma doença.

Os índices de prevalência da IU feminina sofrem variação de acordo com a metodologia adotada em cada estudo. Na população norueguesa, entre mulheres com 20 anos ou mais, a prevalência de IU foi de $25 \%^{(2)}$; no entanto, considerando as mais idosas, entre 80 e 89 anos, estes índices foram de $46 \%{ }^{(3)}$. Em jovens, principalmente aquelas que realizam atividade física intensa como atletas, dançarinas e militares, com idade média de 19,9 a 31,8 anos, os estudos apontaram índices de $28 \%$ a $51 \%{ }^{(4-6)}$.

A IU era apenas um sintoma até 1998 , quando passou a ser considerada uma doença nas Classificações Internacional de Doenças (CID/OMS). Atualmente a International Continence Society (ICS) define IU como perda involuntária de urina que é um problema social ou higiênico ${ }^{(7)}$ valorizando a queixa das pacientes.

Os estudos atuais têm demonstrado uma grande preocupação com a interferência da IU na qualidade de vida dessas mulheres. Os episódios de IU durante as atividades desenvolvidas diariamente são causadores de constrangimento social, disfunção sexual e baixo desempenho profissional. Estas alterações são causas determinantes de isolamento social, estresse, depressão, sentimento de vergonha, condições de incapacidade e baixa auto-estima que resulta em significativa morbidade ${ }^{(1)}$.

A literatura aponta intervenções de enfermagem que podem auxiliar no diagnóstico e controle da perda urinária contribuindo para melhorar a qualidade de vida das mulheres incontinentes ${ }^{(8-11)}$. Exames simples podem ajudar a enfermeira a identificar os fatores de risco para a IU na mulher, assim como uma anamnese de enfermagem detalhada com a realização de uma simples pergunta: você perde urina quando tosse, espirra ou quando sente forte desejo de urinar? Provavelmente, será possível identificar a IU em $80 \%$ a $90 \%$ dos $\operatorname{casos}^{(12)}$ e determinar as intervenções de enfermagem a serem praticadas.

No entanto, a IU tem sido subestimada, freqüentemente negligenciada e não tem recebido considerada atenção dos profissionais ${ }^{(8)}$, muitas vezes por falta de informações, principalmente, sobre os fatores de risco, o que se torna um obstáculo para o seu diagnóstico precoce.

Portanto, este estudo tem como objetivo identificar os principais fatores de risco de IU na mulher apontados na revisão da literatura, na busca de uma melhor compreensão do assunto, visando uma assistência de enfermagem voltada para o diagnóstico, prevenção e tratamento.

\section{MÉTODO}

Realizou-se uma revisão bibliográfica a partir das bases de dados da Literatura Latino-Americana e do Caribe em Ciências da Saúde (LILACS) e Literatura Internacional em Ciências da Saúde (MEDLINE) consultadas através do site da Biblioteca Virtual em Saúde (BVS) da Biblioteca Regional de Medicina (BIREME) e de pesquisa em bibliotecas. Utilizando a base de dados LILACS e as palavras Incontinência $X$ Urinária $X$ Feminina, foram identificados 33 artigos e usando Incontinência $X$ Urinária $X$ Fatores $X$ Risco nenhum artigo foi encontrado. Na base de dados MEDLINE usando as palavras Urinary $X$ Incontinence $X$ Female foram identificados 1.008 artigos e com Urinary $X$ Incontinence $X$ Risk $X$ Factors, 561.

Para a seleção dos artigos foram considerados os seguintes critérios de inclusão: ter sido publicado no período de 1983 a 2003, estar escrito na língua inglesa, espanhola ou portuguesa e abordar temas relacionados à IU na mulher. Foram excluídos os artigos em que o título focalizava IU infantil e masculina, tratamentos clínicos e cirúrgicos na mulher, qualidade de vida e aqueles que não dispunham de resumo.

Em um primeiro momento, os artigos foram selecionados por meio do título e, em seguida, pelos resumos. Foram selecionados 123 artigos que referiam fatores associados ou de risco para IU na mulher.

Os textos, na íntegra, foram obtidos por meio de: Biblioteca Científica Eletrônica em Linha (Scientific Electronic Library Online - Scielo), Biblioteca Digital da Universidade Estadual de Campinas (Unicamp), Comutação Bibliográfica (COMUT) e Biblioteca da Faculdade de Ciências Médicas da Unicamp. Foram localizados 62 artigos.

Utilizando-se os mesmos critérios de inclusão para seleção dos artigos, foram identificados ainda 25 estudos referenciados nos textos inicialmente selecionados. No total, foram localizados, portanto, 87 artigos na língua portuguesa e inglesa.

Após leitura dos textos na íntegra, foram selecionados 38 artigos que serão discutidos no presente estudo. 


\section{RESULTADOS}

Dos 38 textos selecionados apenas três estudos foram realizados na população brasileira. Os principais fatores de risco ou associados à IU apontados são:

- Idade: considerada o principal fator de risco para a IU feminina, afeta significativamente as mais idosas, em geral a partir do climatério/menopausa, com índices de $43 \%$ na faixa etária de 35 a $81 \operatorname{anos}^{(13)}$. Na população geral, a prevalência de IU aumenta com o aumento da idade ${ }^{(2,12,14-16)}$. Alguns dos distúrbios urinários em mulheres mais idosas podem ser causados pela diminuição da capacidade da bexiga, que passa de 500 a $600 \mathrm{ml}$ para 250 a $300 \mathrm{ml}$, contribuindo para o aumento da freqüência urinária e da noctúria(17), pelo baixo nível de estrógeno após a menopausa ${ }^{(18)}$, doenças crônicas e aumento do índice de massa corpórea (IMC) ${ }^{(16)}$.

- Obesidade: a obesidade é um fator que agrava ou contribui para o desenvolvimento da $\mathrm{IU}^{(19)}$ e a prevalência aumenta com o aumento do peso ${ }^{(16)}$. Presume-se que a associação da IU com a obesidade seja conseqüência da alta pressão intra-abdominal provocada, principalmente, pelo aumento de peso na região da cintura-quadril e, conseqüentemente, do aumento da pressão intravesical alterando o mecanismo do trato urinário(20-21). Um estudo(22) relata que, ao exame urodinâmico, foi observada uma elevação na pressão intravesical durante a realização da manobra de Valsava significativamente maior na paciente obesa e, após o emagrecimento, houve um aumento da transmissão passiva da pressão à bexiga e uretra e diminuição da mobilidade da junção uretro-vesical.

- Paridade: a paridade é um dos fatores que mais se procura associar com o desenvolvimento de IU. Comumente a incontinência aparece durante a gravidez e a prevalência se eleva com o aumento da paridade, favorecendo a IU (14-15,20,23-24). Contrariando estes estudos, outros autores $^{(16,25)}$ não encontraram aumento significativo da ocorrência de IU com o aumento da paridade. Muitas mulheres deixaram de apresentar queixa de IU após o parto, não ocorrendo elevação do risco com o aumento do número de partos.

- Tipos de parto: o parto vaginal está associado com o aumento de casos de IU quando comparado com o parto cesáreo, no entanto, o parto vaginal isoladamente não é o causador da IU e sim, quando associado às lesões e traumas do assoalho pélvico ${ }^{(12,21,23,26)}$. Contudo, quando comparado com nulíparas, tanto o parto vaginal como o cesáreo mostraram associação com a $\mathrm{IU}^{(27)}$.

- Uso de anestesia no parto: embora alguns autores relatem a associação entre a IU e o tipo de anestesia durante o parto, este fator tem sido pouco estudado e existem controvérsias. A analgesia epidural foi considerada como um fator de risco por provocar um prolongamento do segundo estágio do trabalho de parto, aumentando o risco de lesão do assoalho pélvico(23), enquanto que outro autor $^{(26)}$ considerou a anestesia epidural como um fator de proteção devido ao relaxamento da musculatura do assoalho pélvico provocado por este tipo de anestesia, prevenindo direta ou indiretamente o assoalho pélvico de trauma no segundo estágio do parto vaginal.

- Peso do recém-nascido: o peso do recém-nascido, tanto durante a gravidez quanto no parto vaginal, influenciam o aumento da prevalência de IU. Este fator pode estar relacionado com o aumento da pressão intra-abdominal, e, conseqüentemente, com o aumento da pressão intravesical. Além disto, existe o risco para lesão do assoalho pélvico durante o parto vaginal ${ }^{(23)}$. Porém, outro estudo não encontrou associação significativa entre o parto de crianças com peso maior que $4000 \mathrm{~g}$ e a presença de $\mathrm{IU}^{(28)}$ ou de lesões no assoalho pélvico de terceiro ou quarto grau após o parto vaginal, fator considerado como risco para $\mathrm{IU}^{(29)}$

- Menopausa: as prevalências de IU em mulheres na pré e pós-menopausa têm sido muito estudadas e os resultados têm confirmado associação significativa, com índices que variam de $46 \%$ a $64 \%^{(3,12,20,23,30)}$. A estática pélvica pode ser afetada com as mudanças hormonais durante a menopausa. O hipoestrogenismo na pós-menopausa predispõe a mulher à $I U^{(12,18)}$ e contribui para sintomas urinários como aumento da freqüência, urgência e disúria(17).

- Cirurgias ginecológicas: há associação significativa entre a IU e cirurgias ginecológicas ${ }^{(30)}$. Alguns autores ${ }^{(15-16,21)}$ observaram achados significativos entre a histe-rectomia e a IU. Acredita-se que a excisão ou o prolápso do útero comprometem as funções do assoalho pélvico, visto que, este órgão suporta parte deste assoalho a sua remoção pode causar danos nas estruturas que sustentam a bexiga e a uretra ${ }^{(16,21)}$. Entretanto, em dois estudos de revisão bibliográfica, mostraram resultados divergentes, pois, um relatou que a histerectomia aumenta a prevalência $I U^{(31)} \mathrm{e}$ outro, não observou aumento significativo de IU após a retirada do útero(32).

- Constipação intestinal: atualmente, a constipação tem sido estudada como um fator que aumenta o risco de IU em mulheres ${ }^{(17,21)}$. A constipação crônica afeta a função urológica: o estiramento do reto pode comprimir a bexiga, contribuindo para a retenção urinária, causando infecção do trato urinário e, freqüentemente, a força realizada durante a evacuação intestinal pode lesar a musculatura pélvica e, através da distensão, traumatizar e causar isquemia muscular ${ }^{(17,21)}$.

- Doenças crônicas: fatores co-existentes como a diabetes e doenças neurológicas são fatores de risco para $\mathrm{IU}^{(10,16,20,23,33)}$. A associação entre o diabetes e a IU mostrou um possível aumento da vulnerabilidade do assoalho pélvico devido a uma mudança do tecido biológico e da 
inervação do músculo pélvico ou devido a lesões nas inervações neuropáticas autônomas da bexiga ou pelo aumento da freqüência urinária causada pela hiperglicemia decorrente do aumento do volume urinário ${ }^{(16,23)}$. As condições neurológicas, incluindo doenças que afetam o movimento, podem causar contração desinibida da bexiga nessas pacientes e a IU será secundária à doença préexistente $\mathrm{e}^{(10,33)}$.

- Fatores hereditários: diferenças significantes de prevalência de IU foram encontradas entre mulheres negras, hispânicas e brancas ${ }^{(34)}$. Quanto aos tipos de IU, a hiperatividade vesical predominou entre mulheres negras e a IU de esforço entre as hispânicas, asiáticas e brancas ${ }^{(35)}$. As mulheres da raça branca têm maior prevalência de IU quando comparadas com as da raça negra, supõe-se que provavelmente existem determinantes genéticos, diferenças na anatomia ou na resistência da uretra e nas estruturas de suporte do assoalho pélvico que protegem as mulheres negras da $\mathrm{IU}^{(20)}$. Ao comparar as mulheres incontinentes com as continentes, a história familiar de IU foi 2,6 vezes maior nas mulheres incontinentes e mais provável ter pelo menos um membro da família com IU ${ }^{(36)}$. No Brasil, não encontraram diferença no risco de IU em relação à raça e, consideraram a possibilidade de que parte das mulheres da amostra estudada poderia ter traços de herança negra. Neste sentido observa-se que, na população brasileira, as distinções raciais com base na cor da pele declarada ou observada não são precisas, dificultando o estabelecimento desta diferença ${ }^{(25)}$.

- Uso de drogas: o uso de medicamentos é fator que contribui para a IU transitória. Alguns medicamentos aumentam a freqüência e a urgência urinária ${ }^{(37-38)}$. Certas drogas, como os simpaticomiméticos e parasimpaticolíticos, atuam no trato urinário inferior e podem alterar a função vesical piorando ou contribuindo para aumento da freqüência da perda urinária.

- Consumo de cafeína: a cafeína tem uma ação diurética nos rins aumentando o volume urinário. A ingestão da cafeína em alta concentração pode causar instabilidade do músculo detrusor e, conseqüentemente, perda involuntária de urina ${ }^{(39-40)}$. Um estudo ${ }^{(40)}$ verificou que a hiperatividade vesical apresentou associação estatisticamente significativa com o consumo de cafeína, idade e tabagismo. A cafeína pode determinar hiperatividade vesical devido ao fato de ter efeito excitante sobre a musculatura lisa do detrusor. Na cistometria ocorre aumento de urgência e freqüência da micção após a ingestão da cafeína; o aumento da pressão na bexiga cheia indica uma diminuição da capacidade vesical, provavelmente sendo a cafeína responsável por este sintoma, podendo não ser um efeito puramente diurético ${ }^{(39)}$. Estes estudos não determinaram se a diminuição da ingestão do café poderia estar associada com a melhora dos sintomas.
- Tabagismo: o fumante freqüentemente apresenta tosse mais violenta, causando efeito direto ou indireto na bexiga ou na uretra podendo danificar os componentes e o mecanismo esfincteriano da uretra propiciando a IU e piorando a freqüência e a intensidade da IU existente ${ }^{(41)}$. As fumantes apresentam aumento significativo na pressão vesical com a tosse, quando comparadas com as não fumantes ${ }^{(42)}$. Os componentes do tabaco (monóxido de carbono, nicotina) causam deficiência no estrógeno, assim, nas fumantes a menopausa natural, fator de risco para IU, ocorre de um a um ano e meio mais cedo ${ }^{(43)}$.

- Exercícios físicos: o exercício físico rigoroso é um dos fatores de risco para a IU em mulheres jovens e nulíparas $^{(4-6)}$. Considerando-se os esportes individuais, a prevalência de IU é maior entre ginastas, porque os exercícios praticados por elas são mais rigorosos na região abdominal em comparação com outros esportes, indicando que a prática da ginástica aumenta a pressão intraabdominal ${ }^{(4-6)}$.

\section{CONCLUSÃO}

A revisão bibliográfica mostrou que os principais fatores de risco para IU na mulher foram: idade, trauma do assoalho pélvico, fatores hereditários, raça, menopausa, obesidade, doenças crônicas, uso de alguns simpaticomiméticos e parasimpaticolíticos, constipação, tabagismo, consumo de cafeína e exercícios intensos na região abdominal.

A literatura apontou vários fatores de risco para IU, entretanto, os resultados dos estudos foram por vezes contraditórios, indicando que de acordo com a metodologia adotada poderão ser identificados diferentes fatores associados à IU na mulher.

No Brasil, poucos trabalhos científicos têm se dedicado a IU, o que dificulta a comparação dos resultados ${ }^{(44)}$. Mediante estes fatos, o reduzido número de estudos referentes aos fatores de risco (ou fatores associados) para IU na mulher brasileira interfere na identificação dos principais fatores que causam a IU na nossa população.

As mulheres incontinentes raramente falam sobre o seu problema e, quando questionadas, muitas vezes procuram omitir por se sentirem constrangidas, por esta mesma razão não procuram tratamento. Entretanto, a IU tende a se acentuar no período pós-menopausa com grau elevado de perda. Amiúde, a não intervenção do profissional de enfermagem refletirá negativamente na realização das atividades de vida diária.

Assim sendo, na assistência à saúde da mulher, se faz necessária a identificação do problema e de seus fatores de risco, bem como a inclusão de intervenções para prevenção, diagnóstico e tratamento relacionados à perda urinária feminina. 
1. Simeonova Z, Milson I, Kullendorff AM, Molander U, Bengtsson $C$. The prevalence of urinary incontinence and its influence on the quality of life in women from urban Swedish population. Acta Obstet Gynecol Scand. 1999;78(6):546-51.

2. Hannestad YS, Rortveit G, Sandvik H, Hunskaar. A community-based epidemiological survey of female urinary incontinence: the Norwegian EPINCONT study. J Clin Epidemiol 2000; 55(11):1150-57.

3. Björnsdóttir LT, Geirsson RT, Jónsson PV. Urinary incontinence and urinary tract infections in octogenarian women. Acta Obstet Gynecol Scand. 1998;77(1):105-9.

4. Nygaard IE, Thompson FL, Svengalis SL, Albrigth JP. Urinary incontinence in elite nulliparous athletes. Obstet Gynecol. 1994;84(2):183-7.

5. Davis G, Sherman R, Wong MF, McClure G, Perez R, Hibbert $M$. Urinary incontinence among female soldiers. Mil Med. 1999;164(3):182-7.

6. Thyssem L, Clevin L, Olsen S, Lose G. Urinary incontinence in elite female athletes and dancers. Int Urogynecol J. 2002;13(1):13-5.

7. Abrams $P$, Cardoso L, Fall M, Griffths $D$, Rosier $P$, Ulmsten $U$, et al. The standardization of terminology of lower urinary tract function: repot from the standardization sub-committee of the international continence society. Urology. 2003;61(1):37-49.

8. O'Brien J, Austin M, Seth P, O'Boyle P. Urinary incontinence: prevalence, need for treatment, and effectiveness of intervention by nurse. BMJ. 1991;303(6813):1308-12.

9. Pearson BD, Kelber S. Urinary incontinence: treatments, intervention, and outcomes. Clin Nurse Spec. 1996;10(4):177-82.

10. Gallo ML, Fallon PJ, Staskin DR. Urinary incontinence: steps to evaluation, diagnosis, and treatment. Nurse Pract. 1997;22(2):21-8.

11. Glashan RQ, Lelis MAS. Fatores de risco associados a incontinência urinaria: é possível modificá-los? Acta Paul Enferm. 1999;12(1):43-7.

12. Butler RN, Maby JI, Montella JM, Young GPH. Urinary incontinence: keys to diagnosis of the older woman. Geriatrics. 1999;54(10):22-30.

13. Guarisi T, Pinto-Neto AM, Pedro AO, Faundes A. Fatores associados à prevalência de sintomas urinários em mulheres climatéricas. Rev Bras Ginecol Obst. 1997; 19: 589-96.

14. Elving LB, Foldspang A, Lam GW, Mommsen S. Descriptive epidemiology of urinary incontinence in 3,100 women age 30-59. Scand J Urol Nephol Suppl. 1989;125:37-43.
15. Milsom I, Ekelund P, Molander U, Arvidsson L, Areskoug B. The influence of age, parity, oral contraception, hysterectomy and menopause on the prevalence of urinary incontinence in women. J Urol. 1993;149(6): 1459-62.

16. Brown JS, Seeley DG, Fong J, Black DM, Ensrud KE, Grady D. Urinary incontinence in older women: who is at risk? Obstet Gynecol. 1996;87(5 Pt 1):715-21.

17. Penn C, Lekan-Rutledge D, Joers AM, Stolley JM, Amhof NV. Assessment of urinary incontinence. J Gerontol Nurs. 1996;22(1):8-19.

18. Fantl JA, Cardozo L, McClisch D. Estrogen therapy in the management of urinary incontinence in postmenopausal women: a meta-analysis. First report of the hormones and urogenital therapy committee. Obstet Gynecol. 1994;83(1):12-8.

19. Santos WN, Feitosa J A, Oliveira FC, Oliveira Filho M, Almeida FML, Medeiros FC. Efeitos da obesidade sobre a função urinária na mulher. RBGO 1994; 16(5):175-8.

20. Brown JS, Grady D, Ouslander JG, Herzog AR, Varner RE, Posner SF. Prevalence of urinary incontinence and associated risk factors in postmenopausal women. Obstet Gynecol. 1999; 94(1):66-70.

21. Moller LA, Lose G, Jorgensen T. Risk factors for lower urinary tract symptoms in women 40 to 60 years of age. Obstet Gynecol. 2000;96(3):446-51.

22. Bump RC, Sugerman J, Fantl A.; McClisch DK. Obesity and lower urinary tract function in women: effect of surgically induced weight loss. Am J Obstet Gynecol. 1992;167(2):392-9.

23. Persson J, Hanssen PW, Rydhstroem H. Obstetric risk factors for stress urinary incontinence: a populationbased study. Obstet Gynecol. 2000;96(3):440-5.

24. Fondspang A, Mommsen S, Lam GW, Elving L. Parity as a correlate of adult female urinary incontinence prevalence. J Epidemiol Community Health 1992;46(6): 595-600.

25. Guarisi T, Pinto Neto AM, Osis MJ, Pedro AO, Costa Paiva LH, Faundes A. Incontinência urinaria entre mulheres climatéricas brasileiras: inquérito domiciliar. Rev Saúde Pública. 2001;35(5):428-35.

26. Dimpfl T, Hesse $U$, Schüssler, B. Incidence and cause of postpartum urinary stress incontinence. Eur J Obstet Gynecol Reprod Biol. 1992;43(1):29-33.

27. Rortveit G, Daltveit AK, Hannestad YS, Hunskaar S. Urinary incontinence after vaginal delivery or cesarean section. N Engl J Med. 2003;348(10):900-7. 
28. Krue $\mathrm{S}$, Jensen $\mathrm{H}$, Agger $\mathrm{AO}$, Rasmussen $\mathrm{KL}$. The influence of infant birth weight on post partum stress incontinence in obese women. Arch Gynecol Obstet. 1997;259(3): 143-45.

29. Moalli PA, Ivy SJ, Meyn LA, Zyczynski HM. Risk factor associated with pelvic floor disorders in women undergoing surgical repair. Obstet Gynecol. 2003;101(5): 869-74.

30. Sein A, Eriksen BC, Hunskaar S. A study of female urinary incontinence in general practice. Demography, medical history and clinical findings. Scand J Urol Nephrol. 1996;30(6):465-71.

31. Brown SJ, Sawaya G, Thom DH, Grady D. Hysterectomy and urinary incontinence: a systematic review. Lancet. 2000;356(9229):535-9.

32. Parazzini $F$, Colli $E$, Origgi $G$, Surace $M$, Bianchi $M$, Benzi $G$, et al. Risk factors for urinary incontinence in women. Eur Urol. 2000;37(6):637-43.

33. Romanzi LJ, Heritz DM, Blaivas JG. Preliminary assessment of the incontinent woman. Urol Clin North Am. 1995;22(3):513-20.

34. Sze EHM, Jones WP, Fergurson JL, Backer CD, Dolezal JM. Prevalence of urinary incontinence symptoms among black, white, and Hispanic women. Obstet Gynecol. 2002; 99(4):572-5.

35. Doung TH, Korn AP. A comparison of urinary incontinence among African American, Asian, Hispanic and white women. Am J Obstet and Gynecol. 2002;184(6):1083-6.
36. Elia G, Bergman J, Dye TD. Familial incontinence of urinary incontinence. Am J Obstet Gynecol. 2002; 187(1): 53-5.

37. McBride RE. Assessing and treating urinary incontinence. Home Healthc Nurse. 1996;14(1): 27-32.

38. Butler RN, Maby JI, Montella JM, Young GGPH. Urinary incontinence: primary care therapies for the older woman. Geriatrics. 1999;54(11):31-44.

39. Creigton SM, Stanton SL. Caffeine: does it affect your bladder? Br J Urol. 1990;66(6):613-4.

40. Arya LA, Myers DL, Jackson ND. Dietary caffeine intake and the risk detrusor instability: a case-control study. Obstet Gynecol. 2000;96(1):85-8.

41. Bump RC, McClisch DK. Cigarette smoking and urinary incontinence in women. Am J Obstet Gynecol. 1992; 167(5):1213-8.

42. Baron JA, La Vecchia C, Levi F. The antestrogenic effect of cigarette smoking in women. Am J Obstet Gynecol. 1990;162(2):502-14.

43. Bump RC, McClisch DK. Cigarette smoking and pure genuine stress incontinence of urine: a comparison of risk factors and determinants between smokers and nonsmokers. Am J Obstet Gynecol. 1994;170(2):579-82.

44. Silva APM, Santos, VLCG. Prevalência da incontinência urinária em adultos e idosos hospitalizados. Rev Esc Enferm USP. 2005;39(1):36-45. 